\title{
Effect of silicon oxide coating on a silicon nitride focusing grating coupler efficiency
}

\author{
Alexey Prokhodtsov ${ }^{1,2, *}$, Alexander Golikov ${ }^{1}$, Pavel $\mathrm{An}^{1,3}$, Vadim Kovalyuk ${ }^{1,3}$, and \\ Gregory Goltsman ${ }^{1,2,3}$ \\ ${ }^{1}$ Moscow State Pedagogical University, 119991 Moscow, Russia \\ ${ }^{2}$ National Research University Higher School of Economics, 101000 Moscow, Russia \\ ${ }^{3}$ Zavoisky Physical-Technical Institute of the Russian Academy of Sciences, 420029 Kazan, Russia
}

\begin{abstract}
The dependence of the efficiency of the focusing grating couplers on the period and filling factor before and after deposition of the upper silicon oxide layer was experimentally studied. The obtained data are of practical importance for tunable integrated-optical devices based on silicon nitride platform.
\end{abstract}

One of the way to create fully tunable circuits on a chip is a waveguide integration with metal microheaters [1]. Such microheaters, on the one hand, should be separated from the main part of the waveguides by a thick dielectric layer (to exclude absorption of the optical mode), and, on the other hand, should be located close enough to waveguide for changing temperature as efficiently as possible. In addition, the deposition of dielectric layers on the waveguide leads to a change of effective refractive index $\left(n_{\text {eff }}\right)$, as well as the transmission spectrum of the focusing grating coupler (FGC) $[3,4]$, which should be taken into account at the design stage of tunable integrated circuits.

The aim of this work is to study a shift in the transmission spectrum of FGC before and after deposition of upper silicon oxide $\left(\mathrm{SiO}_{2}\right)$ layer on the nanophotonic circuit.

At the first stage of the study, a 2D array with two different parameters: grating period in a range of $1-1.2 \mu \mathrm{m}$ and filling factor in a range of $0.5-0.9$ were designed. Each individual structure in the array consisted of two FGCs connected by a waveguide [5]. FGC consists of etched strips of a waveguide material (teeth) of width $L$ and period $\Lambda$. The distance between the FGCs equal to $250 \mu \mathrm{m}$ is selected to match the period of the commercial optical fiber array (OFA) for input/output of light.

At the second stage of the study, the nanophotonic structures by a positive e-beam lithography and plasma chemical etching in a $\mathrm{CHF}_{3}$ atmosphere, based on commercially available wafers with $300 \mathrm{~nm}$ silicon nitride layer on top of buried $3 \mu \mathrm{m}$ silicon oxide were fabricated. In Fig. $1(a, b)$ images of one of the fabricated structures, as well as FGCs from a scanning electron microscope are shown.

At the final stage, the optical transmission of nanophotonic structures was measured in the spectral range of $1510-1620 \mathrm{~nm}$. To do this, the light from a tunable laser source (New Focus, TLB 6600) was connected to the chip using OFA with a polarization controller.

\footnotetext{
* Corresponding author: aprohodcov@ hse.ru
} 

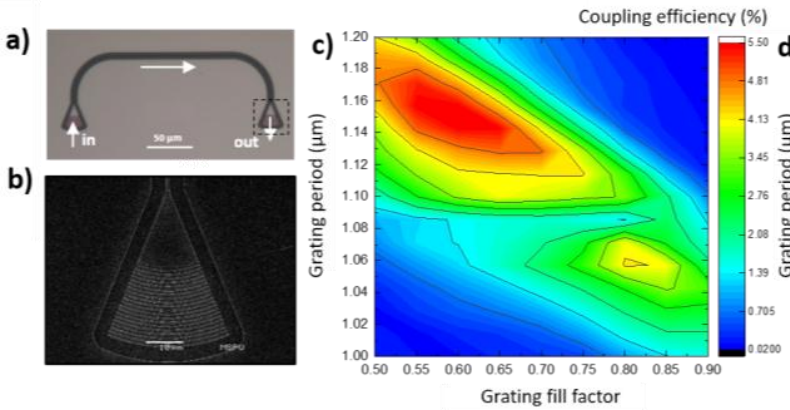

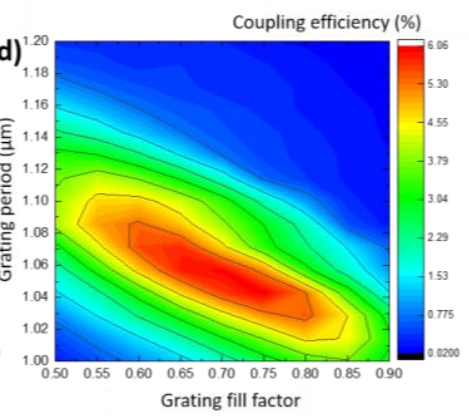

Fig. 1. (a) Image of a fabricated nanophotonic circuit with a waveguide and two FGCs. (b) Image of the FGC obtained by scanning electron microscope. The measured FGC efficiency versus different periods and filling factors: before (c) and after (d) deposition of $\mathrm{SiO}_{2}$

Light passed through on-chip devices and detected at the output by a low-noise photodetector. For preliminary alignment of the chip and OFA, an optical microscope was used, while more precise alignment was achieved by adjusting the position of the motorized $x, y, z$, rotation stage.

Figure $1(\mathrm{c}, \mathrm{d})$ shows the measured contour maps of the coupling efficiency depending on the period and filling factor at a specific wavelength of $1550 \mathrm{~nm}$ before and after the e-beam deposition of $1 \mu \mathrm{m}$ thick $\mathrm{SiO}_{2}$ in vacuum. Due to a change of $n_{\text {eff }}$, a pronounced shift of the central wavelength towards large wavelengths was observed. If the wavelength is fixed at $1550 \mathrm{~nm}$, then an increase in $n_{\text {eff }}$ due to deposition of $\mathrm{SiO}_{2}$ leads to a decrease in the required grating period (Fig. $1 \mathrm{~d}$ ). We associate an increase in the absolute efficiency of FGCs with a decrease of reflection at the air/ $/ \mathrm{SiO}_{2}$ layers compared to more contrast air $/ \mathrm{Si}_{3} \mathrm{~N}_{4}$ optical interface.

Thus, an experimental study of FGCs was carried out with different parameters of the period and filling factor before and after $\mathrm{SiO}_{2}$ deposition. The measured data demonstrate the shift of the central wavelength towards large wavelengths, which is associated with increase of the FGC effective refractive index. The obtained data can be used for development and design of tunable silicon nitride nanophotonic devices with microheaters [1].

This work was supported by the Ministry of Education and Science of the Russian Federation (agreement No. 14.583.21.0065, unique identifier RFMEFI58317X0065).

\section{References}

1. Ovvyan A.P. et al., Journal of Optics 18, 6, 064011 (2016)

2. Marchetti R. et al., Scientific Reports 7, pp. 1 - 8 (2017)

3. Sure A. et al., Opt. Express 11, pp. 3555 - 3561 (2002)

4. Halir R. et al., Opt. Lett. 34, pp. $1408-1410$ (2009)

5. Van Laere F. et al., Opt. Express 19, pp. 1919 - 1921 (2007) 